\title{
HOCHSCHILD DIMENSION OF A SEPARABLY GENERATED FIELD
}

\author{
B. L. OSOFSKY ${ }^{1}$
}

\begin{abstract}
Let $K$ be an $\boldsymbol{\aleph}_{k}$-generated field extension of the field $F$ with transcendence degree $n$. Set $\operatorname{bidim}(K)=$ the projective dimension of $K$ as a $K \otimes_{F} K$-module. Then $K$ locally separably generated implies bidim $(K) \leqq k+n+1$, and $K$ separably generated implies $\operatorname{bidim}(K)=k+n+1$.
\end{abstract}

Let $F$ be a commutative ring, $K$ an $F$-algebra. The Hochschild or bidimension of $K$ over $F$, written $\operatorname{bidim}(K)$, is the projective dimension of $K$ as a module over the ring $R=K \otimes_{F} K^{\text {op }}$. In [3], Hochschild showed that if $K$ is finite over a field $F$, then $\operatorname{bidim}(K)=0$ if and only if $K$ is separable over $F$, i.e., for all field extensions $L$ of $F, K \otimes_{F} L$ is semisimple. Noether [8] had done this for commutative $K$ earlier. Rosenberg and Zelinsky [12] showed $\operatorname{bidim}(K)=0$ implies $[K: F]<\infty$. They also obtained results in the case $K$ is a field relating bidim $(K)$ and the transcendence degree of $K$ over $F$. MacRae [6] took their work and characterized all fields $K$ of bidimension 1 over the field $F$ by showing they are countably generated and so, by Rosenberg and Zelinsky, either separable algebraic extensions of dimension $\boldsymbol{\kappa}_{0}$ or finite separable extensions of rational function fields in one variable. The purpose of this note is to compute the bidimension of any separably generated extension field $K$ of the field $F$. If $K$ is not locally separably generated, Rosenberg and Zelinsky showed $\operatorname{bidim}(K)$ is infinite, but only an upper bound will be obtained in this paper on the locally separably generated case.

Let $\boldsymbol{\aleph}_{-1}$ denote any finite cardinal and $\boldsymbol{\aleph}_{\infty}$ any cardinal $\geqq \boldsymbol{\aleph}_{\omega}$. \#B will denote the cardinality of the set $B$, and $[K: F]$ the dimension of the vector space $K$ over the field $F$. We show the

THEOREM. Let $K$ be a field extension of the field $F$ with separating transcendence basis $B$. Let $[K: F(B)]=\aleph_{m}$. Then $\operatorname{bidim}(K)=\# B+m+1$.

Received by the editors March 7, 1973.

AMS (MOS) subject classifications (1970). Primary 18H20, 13D05; Secondary 16A62.

Key words and phrases. Hochschild dimension of fields, bidimensions of fields, cohomology of algebras.

${ }^{1}$ The author gratefully acknowledges partial support from the NSF under grant GP-32734.

(c) American Mathematical Society 1973 
The method of proof is based on the computation in [10] or [11] of the global dimension of a direct product of fields, but with an appropriate substitute for the "nice sets of idempotents" used in that case.

1. Elementary remarks. Throughout the rest of this paper, $F$ will be a fixed field, and $K$ an extension field of $F$. All fields will be subfields of $K$ containing $F$. $\otimes$ will mean $\otimes_{F}, R$ will denote the ring $K \otimes K$, and $I_{K}$ will denote the kernel of the multiplication map $R \rightarrow K$. Then, since $0 \rightarrow I_{K} \rightarrow R \rightarrow K \rightarrow 0$ is exact, $\operatorname{bidim}(K) \neq 0$ implies $\operatorname{bidim}(K)=$ p.d. $\left(I_{K}\right)+1$. The reader is referred to any standard reference, such as [2] or [5], for the definition of projective dimension. Hence we need only calculate p.d. $\left(I_{K}\right)$ to get our result. We will do that in the algebraic case. Our initial reduction is to that case.

1. Lemma. Let $S$ be any ring, $x$ a central nonunit, nonzero divisor of $S$. Let $S=S / x S$. If $M_{S}$ is an $\bar{S}$-module of finite projective dimension, then p.d. $S_{S}(M)=$ p.d. $S(M)+1$.

Proof. See [4] or perhaps [11].

2. Lemma. Let $x \in K$ be transcendental over $F$. Then

$$
K \otimes_{F^{\prime}} K /(x \otimes 1-1 \otimes x) \simeq K \otimes_{F^{\prime}(x)} K
$$

and $\bar{x}=x \otimes 1-1 \otimes x$ is not a zero divisor in $K \otimes_{F^{\prime}} K=R$.

Proof. The natural map $R \rightarrow K \otimes_{F^{\prime}(x)} K$ has kernel $Z$ generated by

$$
\{u p \otimes v-u \otimes p v \mid u, v \in K, p \in F(x)\} .
$$

But $u p \otimes v-u \otimes p v=(p \otimes 1-1 \otimes p)(u \otimes v)$, so $\{p \otimes 1-1 \otimes p \mid p \in F(x)\}$ generates $Z$. In particular, $\bar{x} \in Z$. Now let $p / q \otimes 1-1 \otimes p / q$ be a generator of $Z$, with $p, q \neq 0 \in F[x]$. Let $\equiv$ denote congruence modulo $(\bar{x})$. Then $x \otimes 1 \equiv 1 \otimes x$, so $p \otimes 1 \equiv 1 \otimes p$ and $q \otimes 1 \equiv 1 \otimes q$. Then $(q \otimes 1)^{-1}=1 / q \otimes 1 \equiv(1 \otimes q)^{-1}=1 \otimes 1 / q$, so $p / q \otimes 1 \equiv 1 \otimes p / q$, and $Z=(\bar{x})$. Let $0 \neq \sum u_{i} \otimes v_{i} \in R$. If $\sum u_{i} \otimes x v_{i}=$ $\sum x u_{i} \otimes v_{i}$, then for all $i, x v_{i}=\sum \alpha_{i j} v_{j}$ with $\alpha_{i j} \in F$, so $\operatorname{det}\left(x I-\left(\alpha_{i j}\right)\right)=0$, contradicting the transcendental property of $x$.

3. Lemma. Let $K$ be a field extension of $F$ with separating transcendence basis $B$ and $[K: F(B)]=\boldsymbol{\aleph}_{m}$. If $\# B=0$ implies bidim $(K)=m+1$, then for any $K, \operatorname{bidim}(K)=\# B+m+1$.

Proof. If both $\# B$ and $m$ are finite, we use induction on $n=\# B$. The basis is assumed. If $\# B>0$, let $x \in B$. Then $K$ is a $K \otimes K /(x \otimes 1-1 \otimes x) \simeq$ $K \otimes_{F(x)} K$-module of projective dimension $(n-1)+m+1$ by the induction hypothesis. Apply Lemma 1. 
If $n=\# B$ or $m$ is infinite, then, given any natural number $k, K$ contains a purely transcendental subfield of degree $k+1$ and hence bidimension $k+1$ by repeated applications of Lemma 1 , or $K$ contains an $\boldsymbol{\aleph}_{k}$-dimensional extension of $F(B)$ which has bidimension $n+k+1$ by the finite case. In either case, since Rosenberg and Zelinsky show in [12] that the bidimension of $K$ is equal to or greater than the bidimension of any subfield, $\operatorname{bidim}(K)=\infty=n+m+1$.

We may thus assume for the rest of this paper that $K$ is a separable algebraic extension of $F$.

4. Remark. Let $L$ be a finite dimensional separable extension of $F$. Then the number of subfields of $L$ containing $F$ is finite. For a proof, pick a book - any book - on classical Galois theory.

5. Corollary. $[K: F]=\boldsymbol{\aleph}_{n}$ implies $K$ contains (at most) $\boldsymbol{\aleph}_{n}$ finite dimensional extensions of $F$.

Proof. $K$ may be expressed as an ascending union of $\boldsymbol{\aleph}_{n}$ smaller dimensional subfields, and every finite extension of $F$ is in one of these. Hence $K$ has at most $\boldsymbol{\aleph}_{n} \cdot \boldsymbol{\aleph}_{n}$ finite dimensional subfields by induction on $n$. It is clear this number is reached.

6. Remark. Let $\alpha$ be any element of $K$. Set $\bar{\alpha}=\alpha \otimes 1-1 \otimes \alpha$. Let $0 \neq x=\sum u_{i} \otimes v_{i}$. The set $\left\{v_{i}\right\}$ will be called the "second coordinates" of $x$. Without loss of generality, these "second coordinates" are linearly independent over $F$. Then $\bar{\alpha} x=0$ implies $\left\{v_{i}\right\} \cup\left\{\alpha v_{i}\right\}$ must be linearly dependent, so there exist $\left\{k_{i}, l_{i}\right\} \subseteq F$ not all zero with $\sum k_{i} v_{i}+\alpha \sum l_{i} v_{i}=0$. Since the $v_{i}$ are linearly independent, $\sum l_{i} v_{i} \neq 0$, so $\alpha=\sum k_{i} v_{i} / \sum l_{i} v_{i} \in F\left[\left\{v_{i}\right\}\right]$.

Now let us look at $I_{K}$. For any finite extension $L$ of $F$ in $K, I_{L}=$ $\operatorname{kernel}(L \otimes L \rightarrow L)$ is generated by an idempotent $e_{L}$ by the NoetherHochschild result mentioned in the introduction ( $L \otimes L \rightarrow L \rightarrow 0$ splits). Any element in $I_{K} \cap L \otimes L$ must lie in $e_{L} R$. This use of $L$ for finite dimensional extensions of $F$ in $K$ and $e_{L}$ for generating idempotents in $I_{L}=$ $I_{K} \cap L \otimes L$ will be maintained throughout the paper, as will the notation $\bar{\alpha}=\alpha \otimes 1-1 \otimes \alpha$ for all $\alpha \in K$.

7. Lemma. Let $I$ be any right ideal of a ring $S$ such that $I$ is generated

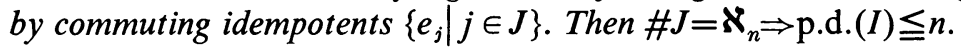

Proof. If $n=0, I=\bigoplus_{i=0}^{\infty} e_{i} \prod_{j=0}^{i-1}\left(1-e_{j}\right) S$ is projective. Auslander's Proposition 1 of [1] and an easy induction complete the proof (see [10] or [11]).

8. Corollary. $[K: F]=\boldsymbol{\aleph}_{n} \Rightarrow$ p.d. $\left(I_{K}\right) \leqq n$.

Proof. Any element $\sum u_{i} \otimes v_{i}$ of $I_{K}$ lies in $L \otimes L$ for some finite dimensional $L$. Hence $\left\{e_{L}\right\}$ generate $I_{K}$. Apply 5 and 7 . 
2. The lower bound. To get a lower bound on the bidimension of $K$, we need a lower bound on the dimension of $I_{K}$, still assuming $K$ is algebraic. The machinery of $[10]$ can be used for getting a projective resolution and direct summand of a projective image in that resolution to apply induction. However, the idempotents generating $I_{K}$ are not "nice" in the sense of [10], so a different basis and induction step are needed. Trying to use induction on the dimension of $K$ over $F$ directly did not seem to work, but led to the following type of ideal which does.

Let $\Lambda$ be a set of finite dimensional fields $L$ directed under $\subseteq$. Let $f$ be an idempotent of $R$ such that, for all $L \in \Lambda, 0 \neq f\left(1-e_{L}\right)$. Set $I_{f}(\Lambda)=$ $\sum_{L \in \Lambda} f e_{L} R$. Note that if $\Lambda$ is the set of all finite dimensional extensions of $F$ in $K$ and $K$ is infinite dimensional, then $\# \Lambda=[K: L], 1 \cdot\left(1-e_{L}\right) \neq 0$ for all $L \in \Lambda$, and $I_{1}(\Lambda)=I_{K}$.

9. Proposition. For any idempotent $f$ and any $\Lambda$ such that for all $L \in \Lambda, 0 \neq f\left(1-e_{L}\right)$, we have

$$
\text { p.d. }\left(I_{f}(\Lambda)\right) \leqq n \Rightarrow \# \Lambda \leqq \aleph_{n} .
$$

Proof. We use induction on $n$. The basis is a modification of an argument in Magid [7]. Let $n=0$, so $I_{f}(\Lambda)$ is projective. By the dual basis lemma (the map $\bigoplus_{L \in \Lambda} R \rightarrow \sum f e_{L} R \rightarrow 0$ splits) there exist maps

$$
\left\{\theta_{L} \in \operatorname{Hom}_{R}\left(I_{f}(\Lambda), R\right) \mid L \in \Lambda\right\}
$$

such that for all $u \in I_{f}(\Lambda), u=\sum_{L} \theta_{L}(u) f e_{L}$ and $\theta_{L}(u)=0$ for almost all $L \in \Lambda$. Since $\theta_{L}$ is an $R$-map, $\theta_{L}(u) f e_{L}=\theta_{L}\left(u f e_{L}\right)=u \theta_{L}\left(f e_{L}\right)$. Set $g_{L}=$ $\theta_{L}\left(f e_{L}\right)$. Then $u g_{L}=0$ for almost all $L$, and $g_{L}=f g_{L}$ for all $L \in \Lambda$.

Case (i). $g_{L}=0$ for almost all $L$. Then $I_{f}(\Lambda)=\sum_{i=1}^{n} f e_{L_{i}} R$, and if $L$ is an element of $\Lambda$ containing each $L_{i}, I_{f}(\Lambda)=e_{L} f R$. Now let $\alpha \in \bigcup_{L \in \Lambda} L$. Then $f \bar{\alpha} \in f e_{L} R$, so $f \bar{\alpha}=f e_{L} \bar{\alpha}$, i.e. $\left(1-e_{L}\right) f \bar{\alpha}=0$. By Remark 6, $\alpha$ must belong to the finite dimensional subfield $L^{\prime}$ generated by "second coordinates" of the nonzero element $\left(1-e_{L}\right) f$. Hence $\bigcup_{L \in \Lambda} L \subseteq L^{\prime}$ which has only finitely many subfields, and so $\# \Lambda<\boldsymbol{\kappa}_{0}$.

Case (ii). There exists a countable set $\left\{L_{i} \mid i \in \omega\right\} \subseteq \Lambda$ such that for all $i \in \omega, g_{L_{i}} \neq 0$. Let $L^{\prime}$ be the subfield of $K$ generated by all "second coordinates" of $\left\{g_{L_{i}} \mid i \in \omega\right\}$. Then $\left[L^{\prime}: F\right]=\aleph_{0}$ so $L^{\prime}$ has only $\aleph_{0}$ finite dimensional subfields by 5 . For all $\alpha \in \bigcup_{L \in \Lambda} L, \bar{\alpha} f g_{L_{i}}=0$ for almost all $i$, so by Remark 6, $\alpha \in L^{\prime}$ and $\bigcup_{L \in \Lambda} L \subseteq L^{\prime}$. Hence $\# \Lambda=\aleph_{0}$.

For $n>0$, the machinery of [10] is available to give our induction step. Since it is written out in reasonable detail in [10] and [11], we just sketch this machinery.

Assume $\# \Lambda>\boldsymbol{\aleph}_{n}$, and linearly order $\Lambda$ in such a way that no ordinal 
$\leqq \aleph_{n}$ is cofinal in $\Lambda$. One gets a projective resolution of $I_{f}(\Lambda)$ by taking

$$
\begin{aligned}
P_{k}(\Lambda) & =\underset{L_{0}<L_{1}<\cdots<L_{k}}{\bigoplus}\left\langle L_{0}, L_{1}, \cdots, L_{k}\right\rangle \prod_{i=0}^{k} e_{L_{i}} f R . \\
d_{k}\left\langle L_{0}, L_{1}, \cdots, L_{k}\right\rangle & =\sum_{i=0}^{k}(-1)^{i}\left\langle L_{0}, \cdots, L_{i-1}, L_{i+1}, \cdots, L_{k}\right\rangle \prod_{j=0}^{k} e_{L j} f . \\
d_{0}\left\langle L_{0}\right\rangle & =e_{L_{0}} f .
\end{aligned}
$$

A check that this creates an acyclic complex uses the easily checked result that, for all $p \in P_{k-1}(\Lambda)$ and for any $M$ greater than all $L$ 's appearing in tuples of $p$,

$$
d_{k}\left(p^{*} M\right)=(-1)^{k} e_{M} p+\left(d_{k-1} p\right)^{*} M
$$

where ${ }^{*} M$ denotes the homomorphism induced on sums of smaller entries by

$$
\left\langle L_{0}, \cdots, L_{k-1}\right\rangle^{*} M=\left\langle L_{0}, \cdots, L_{k-1}, M\right\rangle e_{M} .
$$

Since p.d. $\left(I_{f}(\Lambda)\right)=n, d_{n} P_{n}(\Lambda)$ is projective. Then there exists a directed subset $\Delta \subseteq \Lambda$ such that $\# \Delta=\boldsymbol{\aleph}_{n}$ and $d_{n} P_{n}(\Delta)$ is a direct summand of $d_{n} P_{n}(\Lambda)$. There is a very slight change in the proof of 2.49 of [11] involved here-rephrase as in the proof of 2.53 of that paper to get the directed property. Now look at the subfield $L^{\prime}$ of $K$ generated by all "second coordinates" of $\left\{f\left(1-e_{J}\right) \mid J \in \Delta\right\}$. It is $\boldsymbol{\aleph}_{n}$-generated and so has at most $\aleph_{n}$ finite subfields. Hence there exists $M \in \Lambda, M>J$ for all $J \in \Delta$, such that $M$ is not contained in $L^{\prime}$. Let $\alpha$ generate $M$ over $F$ (every finite dimensional separable extension is generated by a single element). Then $\bar{\alpha} f\left(1-e_{J}\right) \neq 0$ by Remark 6 , and since $\bar{\alpha}=\bar{\alpha} e_{M}, e_{M} f\left(1-e_{J}\right)$ is nonzero for all $J \in \Delta$. Thus we may talk about the ideal $I_{e_{M} f}(\Delta)$.

Using the relation $(*)$ as in [11], one sees that $e_{M} d_{n} P_{n}(\Delta)$ is actually a direct summand of $P_{n-1}(\Lambda)$ and hence of $e_{M} P_{n-1}(\Delta)$. Moreover, $\left\{e_{M} P_{k}(\Delta), d_{k}\right\}$ is a projective resolution of $I_{e_{M} f}(\Delta)$ exactly as $\left\{P_{k}(\Lambda), d_{k}\right\}$ is one of $I_{f}(\Lambda)$. But $d_{n}\left(e_{M} P_{n}(\Delta)\right)$ is a direct summand of $e_{M} P_{n-1}(\Delta)$, so p.d. $\left(I_{e_{M f}}(\Delta)\right) \leqq n-1$ and $\# \Delta=\boldsymbol{\aleph}_{n}$, contradicting the induction hypothesis.

Putting Lemma 3, Corollary 8, and Proposition 9 together, we obtain

10. THEOREM. If $K$ is a separably generated extension field of $F$ with separating transcendence basis $B$, such that $[K: F(B)]=\aleph_{m}$ then $\operatorname{bidim}(K)=$ $\# B+m+1$.

Proof. If $K$ is separably algebraic, apply induction on $m$. For $m=-1$, this is the Noether-Hochschild result. If $m \geqq 0$,

$$
\operatorname{bidim}(K)=1+\text { p.d. }\left(I_{1}(\text { all finite extensions of } F \text { in } K)\right) \geqq 1+m
$$

by 9 and $\leqq 1+m$ by 8 . Lemma 3 finishes the proof. 
3. An upper bound in the locally separably generated case. The reduction to the algebraic case to obtain a lower bound used in $\S \S 1$ and 2 does not work in the case that $K$ is only locally separably generated but not separably generated. Rosenberg and Zelinsky's example where $K=$ $\bigcup_{n=0}^{\infty} F\left(x^{1 / p^{n}}\right), x$ transcendental and $F$ of characteristic $p$, clearly illustrates this. Yet we can still get an upper bound as in the separably generated case by using Auslander's proposition in [1] to extend the argument for $\boldsymbol{\aleph}_{\mathbf{0}}$ in [12].

11. Lemma. Let $K^{\prime}$ be a finitely generated, separably generated subfield of $K$. Then p.d. ${ }_{K^{\prime} \otimes K^{\prime}}\left(I_{K^{\prime}}\right)=$ p.d. ${ }_{R}\left(I_{K^{\prime}} R\right)$.

Proof. We use induction on transcendence degree $K^{\prime}=n$. If $n=0, I_{K}$, is generated by an idempotent, and so is $I_{K^{\prime}} R$, so both are projective. If $n>0$, let $x$ be an element of a separating transcendence basis for $K^{\prime}$. Then $(\bar{x})$ is projective in $K^{\prime} \otimes K^{\prime}$ or $K \otimes K$ but not a direct summand, so for both rings, p.d. $\left(\tilde{I}_{K^{\prime}} /(\bar{x})\right)=$ p.d. $\left(\tilde{I}_{K^{\prime}}\right)$ (or p.d. $\left(\tilde{I}_{K^{\prime}}\right)+1$ if $n=1$ ), where $\tilde{I}_{K^{\prime}}$ is the appropriate one of $I_{K^{\prime}}$ or $I_{K^{\prime}} R$. Since $R /(\bar{x}) \simeq K \otimes_{F(x)} K$ and $I_{K^{\prime}} /(\bar{x})$ is the kernel of the multiplication map from $K^{\prime} \otimes_{F^{\prime}(x)} K^{\prime} \rightarrow K^{\prime}$, the result follows from the induction hypothesis and Lemma 1 .

12. Corollary. Let $K$ be locally separably generated, $\operatorname{tr} \operatorname{deg}(K)=n$, $K \boldsymbol{\aleph}_{k}$-generated. Then $\operatorname{bidim}(K) \leqq n+k+1$.

Proof. The finitely generated separably generated subfields $K^{\prime}$ of $K$ form a directed set under $\subseteq$, and there are $\boldsymbol{\aleph}_{k}$ of them. Then $\left\{I_{K^{\prime}} R\right\}$ is a directed system of submodules of $I_{K}$, each of projective dimension $\leqq n$. By [11, Proposition 2.43], p.d. $\left(\sum I_{K^{\prime}} R\right) \leqq n+k+1$. Since $K$ is locally separably generated, $\sum I_{K^{\prime}} R=I_{K^{*}}$.

\section{BIBLIOGRAPHY}

1. M. Auslander, On the dimension of modules and algebras. III: global dimension, Nagoya Math. J. 9 (1955), 67-77. MR 17, 579.

2. H. Cartan and S. Eilenberg, Homological algebra, Princeton Univ. Press, Princeton, N.J., 1956. MR 17, 1040.

3. G. Hochschild, On the cohomology groups of an associative algebra, Ann. of Math. (2) 46 (1945), 58-67. MR 6, 114.

4. I. Kaplansky, Commutative rings, Queen Mary College Lecture Notes, London, 1966.

5. S. Mac Lane, Homology, Die Grundlehren der math. Wissenschaften, Band 114, Academic Press, New York; Springer-Verlag, Berlin, 1963. MR 28 \#122.

6. R. MacRae, On cardinality, cohomology, and a conjecture of Rosenberg and Zelinsky, Trans. Amer. Math. Soc. 118 (1965), 243-246. MR 31 \#2297.

7. A. Magid, Commutative algebras of Hochschild dimension one, Proc. Amer. Math. Soc. 24 (1970), 530-532. MR 40 \#4259. 
8. E. Noether, Idealdifferentiation und Differente, J. Reine Angew. Math. 188 (1950), 1-21. MR 12, 388.

9. B. Osofsky, Homological dimension and the continuum hypothesis, Trans. Amer. Math. Soc. 132 (1968), 217-230. MR 37 \#205.

10. - Homological dimension and cardinality, Trans. Amer. Math. Soc. 151 (1970), 641-649. MR 42 \#321.

11. - Homological dimensions of modules, CBMS Regional Series in Math., Amer. Math. Soc., Providence, R.I., 1973.

12. A. Rosenberg and D. Zelinsky, Cohomology of infinite algebras, Trans. Amer. Math. Soc. 82 (1956), 85-98. MR 17, 1181.

Department of Mathematics, Rutgers University, New Brunswick, New JERSEY 08903 